\title{
Pion-kaon femtoscopy in Au+Au collisions at STAR
}

\author{
Katarzyna Poniatowska ${ }^{1, a}$, on behalf of the STAR collaboration \\ ${ }^{1}$ Warsaw University of Technology, Koszykowa 75, 00-662 Warsaw, Poland
}

\begin{abstract}
Femtoscopy analysis allows us to extract information about the properties of particle emission source created after collision. From HBT of two correlated pions one can calculate source sizes; in addition, from the non-identical particle correlations, e.g. pion-kaon femtoscopy, one can obtain information not only about source sizes but the asymmetry in the emission processes of particles of different types as well. Such asymmetry gives knowledge of which kind of particles are emitted first/second and/or from which region of the source. The studies of non-identical particle femtoscopy for different collision energies gives us the opportunity to study how the source size and asymmetry in particle emission depend on the initial conditions of the collision. In these proceedings, we will present STAR results of pion-kaon femtoscopy at mid-rapidity in $A u+A u$ collisions from the Beam Energy Scan program.
\end{abstract}

\section{Introduction}

During the Beam Energy Scan (BES) program, the STAR (Solenoidal Tracker at RHIC) experiment collected data at different $A u+A u$ collision energies $\sqrt{s_{N N}}=7.7,11.5,14.5,19.6,27,39 \mathrm{GeV}$. The analysis of data for different collision energies gives the opportunity to study features of the created source as the function of $\sqrt{s_{N N}}$. One of these studies is the femtoscopic analysis of non-identical particles, e.g., pions and kaons [1].

The following work will present the first results of pion-kaon correlations at center-of-mass energies 7.7 and $39 \mathrm{GeV}$. Results for energy $\sqrt{s_{N N}}=39 \mathrm{GeV}$ are presented for the most central collisions (up to $10 \%$ of the total hadronic cross section of the collision) and minimum-bias collisions (0-80\%). Results for $\sqrt{s_{N N}}=7.7 \mathrm{GeV}$ are for data from minimum-bias collisions $(0-80 \%)$. The results are compared with data at $\sqrt{s_{N N}}=130 \mathrm{GeV}[2]$.

\section{Femtoscopy of non-identical particles}

In the case of identical particles, the correlation between them is caused by quantum statistical effects [3]. In the case of non-identical particle correlations the effect occurs due to final state interactions (FSI) - Coulomb force and/or strong interactions. Pion-kaon femtoscopy is dominated by the Coulomb force. Shape of the correlation function (CF) shows us attraction or repulsion between two particles, which depends on the charges of correlated particles.

\footnotetext{
ae-mail: poniatowska@if.pw.edu.pl
} 
The correlation function is given by

$$
C\left(p_{1}, p_{2}\right)=\frac{P_{2}\left(\overrightarrow{p_{1}}, \overrightarrow{p_{2}}\right)}{P_{1}\left(\overrightarrow{p_{1}}\right) P_{1}\left(\overrightarrow{p_{2}}\right)},
$$

where: $P_{2}\left(\overrightarrow{p_{1}}, \overrightarrow{p_{2}}\right)$ is the probability of observing two particles with momentum $\overrightarrow{p_{1}}$ and $\overrightarrow{p_{2}}$ simultaneously; $P_{1}\left(\overrightarrow{p_{1}}\right), P_{1}\left(\overrightarrow{p_{2}}\right)$ are the probabilities of observing these two particles separately.

The aim of this analysis is to investigate the space-time asymmetry in the emission process [2]. The technique used here assumes that the correlation functions are calculated in two groups of pairs [1]. The first group represents the case where the pions catch up with the kaons, and the second case is when the pions move away from the kaons. The criterion of division depends upon the space-time separation between pion and kaon emission points. Each sample is used to construct two different correlation functions, $C_{+}\left(k^{*}\right)$ and $C_{-}\left(k^{*}\right)$, the sign index reflecting the sign of $\vec{v} \cdot \vec{k}_{\pi}^{*}$, with $\vec{v}$ the pair velocity and $\vec{k}_{\pi}^{*}$ the pion momentum vector in the PRF (pair rest frame) [4]. If the average spacetime emission points of pions and kaons coincide, then the two correlation functions are identical. If instead the pions are emitted closer to the center of the source than kaons, then pions with a larger velocity will tend to catch up with the kaons, and the Coulomb correlation strength will be enhanced compared with the case where the pions are slower than the kaons. Hence, the correlation function $C_{+}$will show a larger deviation from unity than $C_{-}$.

Another condition for the separation of pairs can be the sign of $k_{\text {side }}, k_{\text {long }}$ and $k_{\text {out }}$, the $\vec{k}_{\pi}^{*}$ projections onto three perpendicular axes in the longitudinally comoving system (LCMS) where the longitudinal component of the pair momentum vanishes [5]. In the LCMS, the out axis is parallel to the pair velocity, the long axis is the beam axis and the side axis is perpendicular to the other two. The corresponding projections of the three-vector $\vec{r}^{*}$, the relative distance between the particle freeze-out points in the PRF are $r_{\text {out }}^{*}, r_{\text {side }}^{*}$, and $r_{\text {long }}^{*}$. Due to azimuthal symmetry and symmetry about mid - rapidity: $\left\langle r_{\text {side }}\right\rangle=\left\langle r_{\text {long }}\right\rangle=0$. Thus $C_{+} / C_{-}$("double ratio" ), defined with respect to the signs of $k_{\text {side }}^{*}$ and $k_{\text {long }}^{*}$, should not deviate from unity.

\subsection{Data selection}

Table 1 shows the cuts used in the selection of pions and kaons at $\sqrt{s_{N N}}=7.7$ and $\sqrt{s_{N N}}=39 \mathrm{GeV}$, using information from the Time Projection Chamber (TPC) and the time-of-flight (TOF) detector [6][7]. In order to increase the purity of identification, particle with momentum greater than 0.55 $\mathrm{GeV} / \mathrm{c}$ must be registered by both detectors. During the selection of particles registered in the TPC detector, we used particles that were within 3 standard deviation $(\mathrm{N} \sigma)$ of the Bichsel function. To analyse the primary particles, we selected tracks with the distance of closest approach (DCA) to the primary vertex less than $3 \mathrm{~cm}$. Results presented include statistical errors only. The results at $\sqrt{s_{N N}}=130 \mathrm{GeV}$ were obtained using only the TPC for particle identification [2].

\subsection{The comparison of correlation functions for $A u+A u$ collisions at 7.7 and $39 \mathrm{GeV}$}

The magnitude of the correlation effect for $\sqrt{s_{N N}}=39 \mathrm{GeV}$ (Fig. 1) is stronger than for $\sqrt{s_{N N}}=130$ GeV (Fig. 2 a) and b)). Such differences are observed for all correlated systems (particles of the same-sign: $\pi^{+} K^{+}, \pi^{-} K^{-}$; particles of the opposite-sign: $\pi^{+} K^{-}, \pi^{-} K^{+}$). From this difference we can conclude, that the particle emission sources are bigger at center-of-mass energies $130 \mathrm{GeV}$ than 39 $\mathrm{GeV}$. The correlation signal for the same-charge particles $\left(\pi^{+} K^{+}, \pi^{-} K^{-}\right)$and opposite-charge particles $\left(\pi^{+} K^{-}, \pi^{-} K^{+}\right)$are similar. In order to increase the statistics it is possible to merge such pairs (Fig. 2). The CF signals for $\sqrt{s_{N N}}=39 \mathrm{GeV}$ and $\sqrt{s_{N N}}=7.7 \mathrm{GeV}$ are consistent with each other within error 


\begin{tabular}{|c||c|c|}
\hline & $7.7 \mathrm{GeV}$ & $39 \mathrm{GeV}$ \\
\hline \hline analyzed & $3.4 \mathrm{M}$ events & $91.2 \mathrm{M}$ events \\
\hline$\pi$ momentum & {$[0.1,1.2] \mathrm{GeV} / \mathrm{c}$} & {$[0.15,0.7] \mathrm{GeV} / \mathrm{c}$} \\
\hline $\mathrm{K}$ momentum & {$[0.1,1.2] \mathrm{GeV} / \mathrm{c}$} & {$[0.3,0.8] \mathrm{GeV} / \mathrm{c}$} \\
\hline $\mathrm{N} \sigma$ for $\pi$ and $\mathrm{K}$ & $<3.0$ & $<3.0$ \\
\hline$\pi m^{2}$ & {$[0.0176,0.022] \mathrm{GeV}^{2} / \mathrm{c}^{4}$} & {$[0.017,0.026] \mathrm{GeV}^{2} / \mathrm{c}^{4}$} \\
\hline $\mathrm{K} m^{2}$ & {$[0.23,0.26] \mathrm{GeV}^{2} / \mathrm{c}^{4}$} & {$[0.22,0.27] \mathrm{GeV}^{2} / \mathrm{c}^{4}$} \\
\hline$|\eta|$ & $<0.5$ & $<0.5$ \\
\hline $\mathrm{DCA}$ & $<3 \mathrm{~cm}$ & $<3 \mathrm{~cm}$ \\
\hline
\end{tabular}

Table 1. The cuts used in the analysis at $\sqrt{s_{N N}}=7.7$ and $39 \mathrm{GeV}$. The TPC particle identification cuts for these two energies are different.

bars. This simillarity means that the source created in collisions at energy $\sqrt{s_{N N}}=7.7 \mathrm{GeV}$ and 39 $\mathrm{GeV}$ have the same size - taking into account the statistical errors. Work on the estimation of the source sizes is still ongoing.

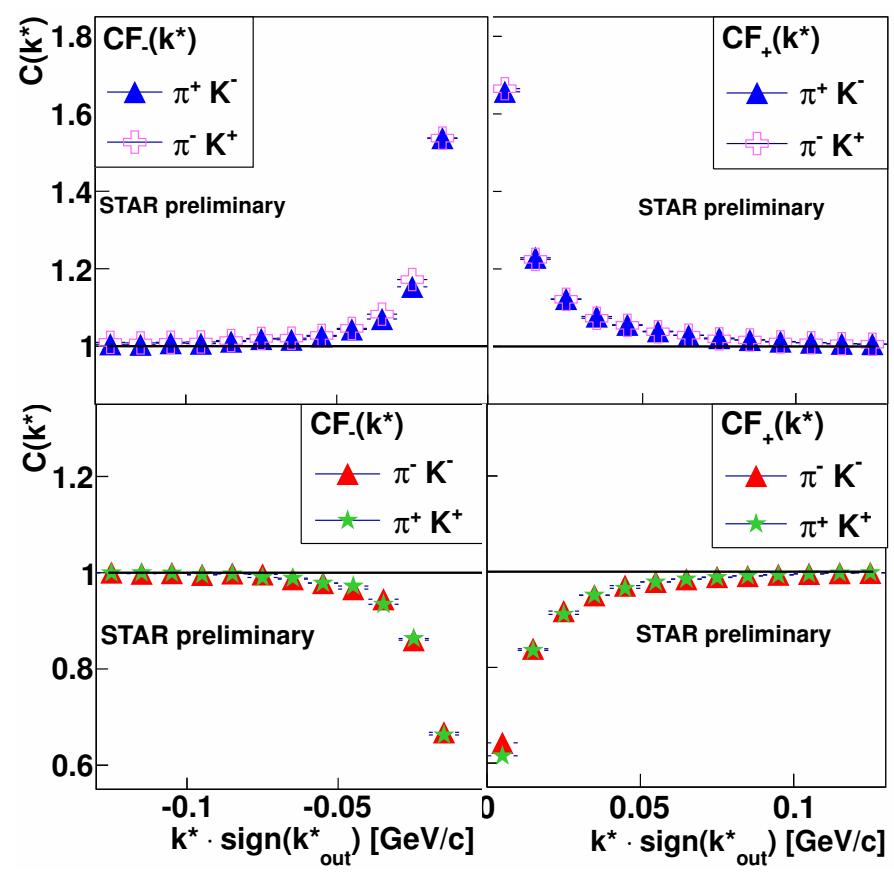

Figure 1. The pion-kaon correlation functions for four different particle combinations $\left(\pi^{+} K^{+}, \pi^{-} K^{-}, \pi^{+} K^{-}, \pi^{-} K^{+}\right)$. Analysis prepared for central collisions $(0-10 \%)$ at $\sqrt{s_{N N}}=39 \mathrm{GeV}$. 


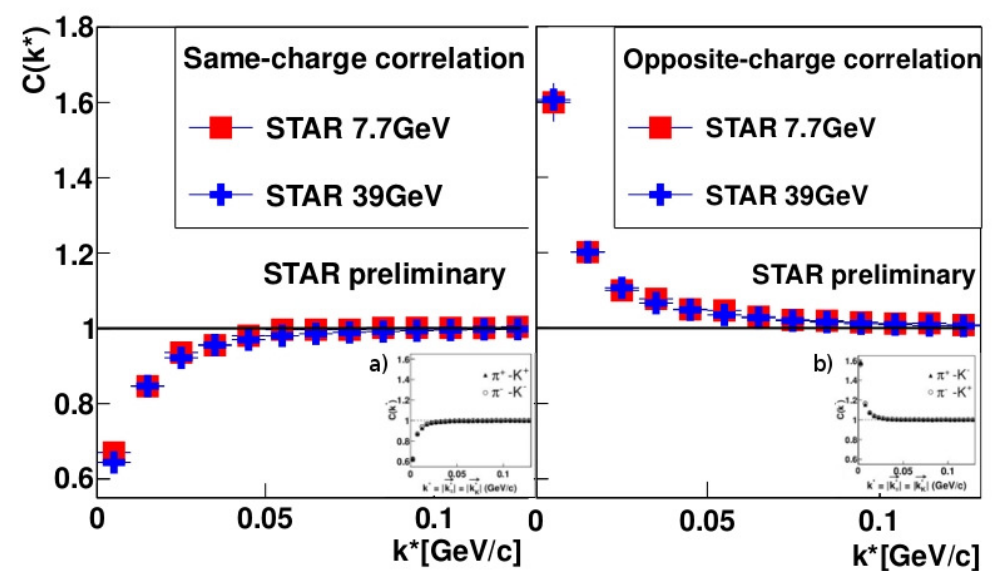

Figure 2. The comparison of pion-kaon correlation functions for $A u+A u$ collisions at $\sqrt{s_{N N}}=39 \mathrm{GeV}$ and $\sqrt{s_{N N}}=7.7 \mathrm{GeV}$ for the minimum-bias collisions $(0-80 \%)$. Results are presented for two types of correlations: same-charge correlation $\left(\pi^{+} K^{+}+\pi^{-} K^{-}\right)$; opposite-charge correlation $\left(\pi^{+} K^{-}+\pi^{-} K^{+}\right)$. On small plots are presented results for $A u+A u$ central $(0-12 \%)$ collisions at $\sqrt{s_{N N}}=130 \mathrm{GeV}$ [2]: a) $\pi^{+} K^{+}$and $\pi^{-} K^{-}$, b) $\pi^{+} K^{-}$and $\pi^{+} K^{-}$.

\subsection{The asymmetry for $A u+A u$ collisions at $\sqrt{s_{N N}}=39 \mathrm{GeV}$ and $7.7 \mathrm{GeV}$}

To test the pion-kaon asymmetry, the "double ratio" function are calculated for $\sqrt{s_{N N}}=39 \mathrm{GeV}$ (Fig. 3), and then the results are compared with those at $\sqrt{s_{N N}}=130 \mathrm{GeV}$ (Fig. 3 parts: a, b, c and d). The magnitude of the correlation effect for the "double ratio" function is stronger for $\sqrt{s_{N N}}=39$ $\mathrm{GeV}$ than for $130 \mathrm{GeV}$. Further investigation is still underway.

Within the larger uncertainties at $\sqrt{s_{N N}}=7.7 \mathrm{GeV}$, the "double ratio" functions at $\sqrt{s_{N N}}=7.7$ and $39 \mathrm{GeV}$ agree well and show similar trends (Fig. 4) .

\section{Summary}

In these proceedings we present correlation functions and the "double ratio" functions for minimumbias and central $A u+A u$ collisions at $\sqrt{s_{N N}}=39 \mathrm{GeV}$ and minimum-bias data $\sqrt{s_{N N}}=7.7 \mathrm{GeV}$. Comparing data from the BES program with data for $\sqrt{s_{N N}}=130 \mathrm{GeV}$ we can conclude that the source created in $A u+A u$ collisions at lower energies is smaller than at higher collision energies. Within present errors, the correlation functions for $\sqrt{s_{N N}}=7.7$ and $39 \mathrm{GeV}$ agree well.

The deviation from the unity of $C_{+} / C_{-}$functions for energies $\sqrt{s_{N N}}=7.7$ and $39 \mathrm{GeV}$ means that the asymmetry in emission process of pions and kaons is observed. The shape of the "double ratio" function indicates that the most of the pions are emitted closer to the system's center or/and later than most of the kaons in systems created in these collisions. The work to estimate the exact parameter describing source size and asymmetry in the emission process between non-identical particles is under way.

\section{Acknowledgements}

This work was supported by the Grants: UMO-2012/07/D/ST2/02123 and supported by National Science Centre, Poland. 


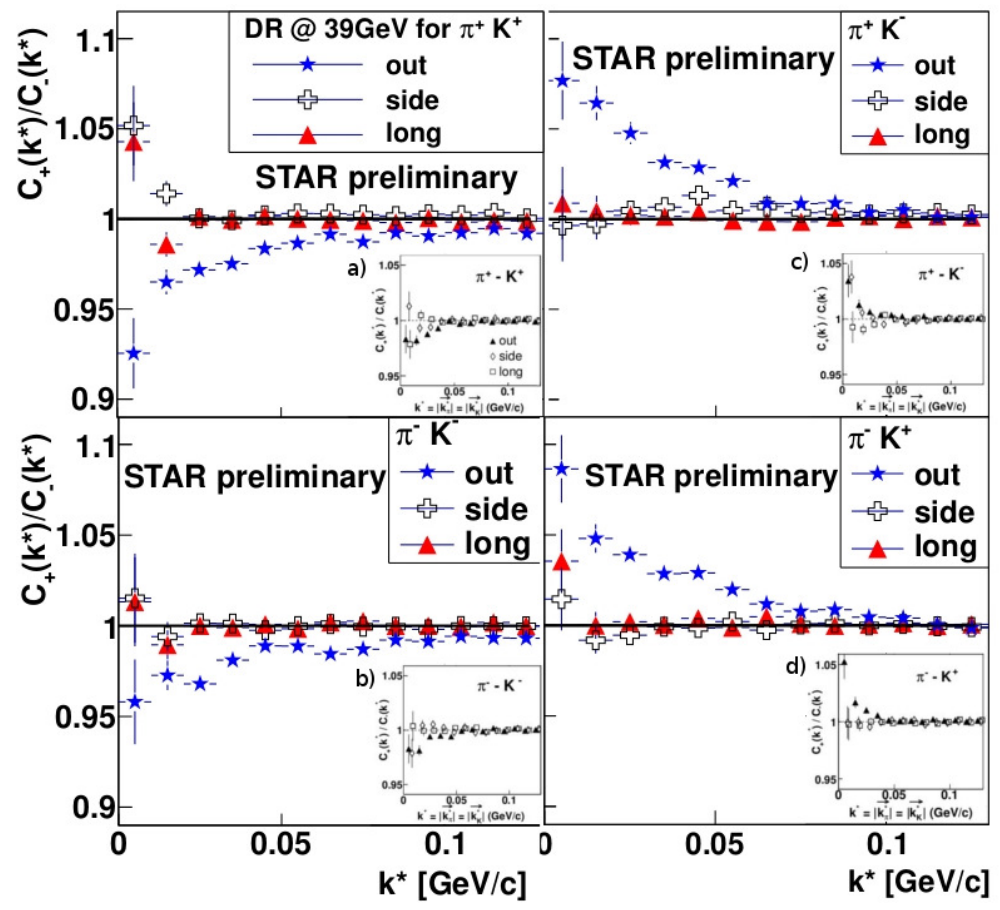

Figure 3. The pion-kaon "double ratio" functions for $A u+A u$ central $(0-10 \%)$ collisions at $\sqrt{s_{N N}}=$ $39 \mathrm{GeV}$ for out, side and long directions. Results are presented for four different particles systems $\left(\pi^{+} K^{+}, \pi^{-} K^{-}, \pi^{+} K^{-}, \pi^{-} K^{+}\right)$. In small charts are results for $A u+A u$ central $(0-12 \%)$ collisions at $\sqrt{s_{N N}}=130 \mathrm{GeV}$ [2]: a) $\pi^{+} K^{+}$, b) $\pi^{-} K^{-}$, c) system $\pi^{+} K^{-}$, d) system $\pi^{+} K^{-}$.

\section{References}

[1] R. Lednicky, V.L. Lyuboshitz, B. Erazmus, D. Nouais, Physics Letters B 373, 30-34, 1996

[2] STAR Collaboration, Phys. Rev. Lett. 91, 262302, 2003

[3] R. Lednicky, Nucl. Phys. A774 189-198, 2006

[4] A. Kisiel, Studies of non-identical meson-meson correlation at low relative velocities in relativistic heavy-ion collisions registered in the STAR experiment, $\mathrm{PhD}$ Thesis, Warsaw Univeristy of Technology, Dec 2004

[5] R. Lednicky, Proc. 8th Int. Workshop on Multiparticle production, Correlations and Fluctuations '98, p. 148-155, 1998

[6] M. Anderson et al.,[STAR Collaboration], Nucl. Instrum. Meth. A 499, 659 (2003)

[7] STAR Time-of-Flight Proposal: http://www.star.bnl.gov/STAR/tof/publications/TOF 20040524.pdf. 
EPJ Web of Conferences

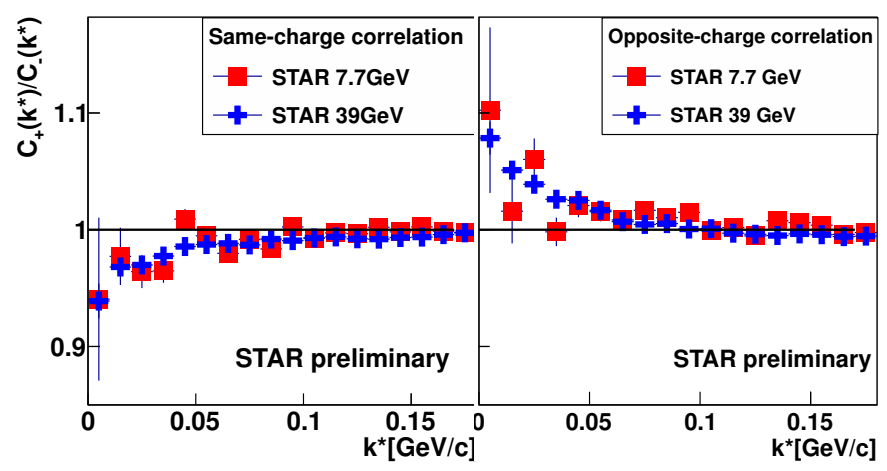

Figure 4. Comparison of "double ratio" functions for pions and kaons for $A u+A u$ minimum-bias $(0-80 \%)$ at $\sqrt{s_{N N}}=39 \mathrm{GeV}$ and $\sqrt{s_{N N}}=7.7 \mathrm{GeV}$ for out direction. Results are presented for two types of correlations: same-charge correlation $\left(\pi^{+} K^{+}+\pi^{-} K^{-}\right)$; opposite-charge correlation $\left(\pi^{+} K^{-}+\pi^{-} K^{+}\right)$. 